\title{
SISTEMA DEL BIOXIDO DE CARBONO EN EL GOLFO DE CALIFORNIA
}

por:

\section{GILBERTO GAXIOLA CASTRO}

\section{SAUL ALVAREZ BORREGO}

Centro de Investigación Cientifica $\mathrm{y}$ de Educación Superior de Ensenada, B. C. Espinoza 843, Ensenada, B.C. México

RICHARD A. SCHWARTZLOSE

Scripps Institution of Oceanography

La Jolla, California 92037 U. S. A.

RESUMEN,

El Golfo de California puede dividirse en dos partes hidrográficas claramente diferenciadas: una al norte del umbral sur de cuenca Salsipuedes, y otra al sur del mismo. En la parte norte las fuertes corrientes de marea provocan una mayor homogeneización de la columa de agua. Cuenca Salsipuedes, y en general todas las cuencas del Golfo, tienden a tener los mismos valores de las propiedades del agua en sus zonas profundas, que Ios encontrados en las profundidades de los umbra les. La parte sur del Golfo tiene distribuciones de las diferentes propiedades del agua muy simila res a las encontradas en océano abierto. Mienträs que la parte norte muestra características únicas. En la parte norte no existen mínimos de oxígeno y salinidad a profundidades intermedias. A $800 \mathrm{~m}$, cuando se comparan la cuenca Salsipuedes (o canal de Ballenas) y la parte sur del Golfo, se halla que la cuenca Salsipuedes tiene aproximadamente $5.5^{\circ} \mathrm{C}, 0.3 \%, 1.3 \mathrm{ml} / 1$ de $0_{2}, 0.18$ unidades de $\mathrm{pH}, 0.1 \mathrm{mM} \mathrm{Kgr}-1$ de bióxido de carbono total, 20 unidades de porciento de saturación de carbonato 
de calcio con respecto a aragonita y 30 unidades de porciento con respecto a calcita, más que la parte sur del Golfo. Debido a los efectos de mezcla por las fuertes corrientes de marea, las aguas superficiales del canal de Ballenas tienen valores mínimos de temperatura, salinidad, oxígeno, $\mathrm{pH}$ y porciento de saturación de carbonato de calcio con respecto a aragonita y calcita, y máximos valores de bióxido de carbono total, con respecto a los valores superficiales del resto del Golfo. Las aguas del Golfo estain sobresaturadas de carbo nato de calcio con respecto a aragonita, de la superficie a 50-150 m, Ilegando a alcanzar 40\% de saturación por debajo de $1000 \mathrm{~m}$ en la parte sur. Con respecto a calcita, en la parte sur del Golfo, los valores de saturación calculados con las ecuaciones de Edmond y Gieskes (1970) muestran sobresaturación de la superficie a $150-300 \mathrm{~m}$, alcanzando $60 \%$ por debajo de $1500 \mathrm{~m}$; y los valores calculados con las ecuaciones de Ingle (1975) muestran sobresaturación de la superficie a 350$600 \mathrm{~m}$, alcanzando $60 \%$ a $3500 \mathrm{~m}$.

\section{ABSTRACT}

The Gulf of California may be divided in two hydrographic regions clearly differentiated: one north from the southern sill of Salsipuedes basin, and the other to the south. In the northern region, the strong tidal currents cause a greater homogeneization of the water column. Salsipuedes basin, and all the basins of the Gulf, tend to have the same values of the water properties at their bottoms as those of sill depths. The southern region of the Gulf has distributions of the different water properties very similar to those found in the open ocean. The northern region shows unique characteristics. There are no oxygen and salinity minima at intermediate depths in the northern region. At $800 \mathrm{~m}$, when Salsipuedes basin (or Ballenas channel) and the southern region of the Gulf are compared, it is found that the Salsipuedes basin has approximately $5.5^{\circ} \mathrm{C}, 0.3 \%$, $1.3 \mathrm{ml} / 1$ of $\mathrm{O}_{2}, 0.18$ of $\mathrm{pH}$ unit, $0.1 \mathrm{mM} \mathrm{Kgr}-1$ of total carbon dioxide, 20 percent units of calcium carbonate saturation with respect to aragonite and 30 percent units of saturation with respect to calcite, more than the southern region. Mixing due to tidal currents cause minimum surface values of temperature, salinity, oxygen, $\mathrm{pH}$ and percent saturation of calcium carbonate with respect to aragonite and calcite, and maximum surface values 
of total carbon dioxide at Ballenas channel, with respect to surface values of the rest of the Gulf. The Gulf waters are supersaturated with calcium carbonate, with respect to aragonite, from the surface to $50-150 \mathrm{~m}$; with $40 \%$ saturation in waters deeper than $1000 \mathrm{~m}$, at the southern region. At the southern region, the saturation values with respect to calcite, calculated with Edmond and Gieskes' (1970) equations, for the calcium carbonate solubility product, show supersaturation from the surface to $150-300 \mathrm{~m}$, with $60 \%$ in waters deeper that $1500 \mathrm{~m}$; and the values calculated with Ingle's (1975) equations show supersaturation from the surface to $350-600 \mathrm{~m}$, with $60 \%$ at $3500 \mathrm{~m}$.

\section{INTRODUCCION,}

El carbono es un elemento biológicamente activo, Su distribución en el océano es influenciada por consumo biológico en las aguas superficiales y por la oxidación de materia orgántca y la solución de carbonatos a profundidades mayores. El carbono es un nutriente y su distribución tiende a seguir la de otros nutrientes como el fosfato, nitrato y silicato. Sin embargo, hay diferencias importantes entre la distribución del carbono $y$ la de los otros nutrientes. Una es que el carbono inorgánico está presente en exceso y nunca limita el crecimiento de las plantas. Otra es que la distribución superficial del carbono inorgánico está afectada por el intercambio con la atmósfera (Culberson, 1972).

No existía hasta ahora ninguna publicación que describiera el sistema del bióxido de carbono en el Golfo de California. Por ello, se hicieron determinaciones de $\mathrm{pH}$ y alcalinidad en el crucero del "B/I Alexander Agassiz" del 8 de abril al 4 de mayo de 1974. Este fue un crucero conjunto entre la Institución Scripps de Oceanografía de la Universidad de California y Ia Unidad de Ciencias Marinas de la Universidad Autónoma de Baja California. Los objetivos que se persiguen en el presente escrito son: describir las condictones hidrológicas de primavera en el Golfo de California, incluyendo la distribución vertical de temperatura, salinidad y oxígeno disuelto; $y$ describir la distribución espacial de variables pertinentes al slstema del bióxido de carbono, tales como el pH "in sttu",

alcalinidad total y específica, bibxido de carbono total y porcentajes de saturación de carbonato le calcio con respecto a calcita $\mathrm{y}$ aragonita. 
METODOS Y MATERIALES.

De las veinte estaciones hidrográficas muestreadas en el Golfo de California durante el menctonado crucero, se escogieron quince para los propósitos del presente trabajo. Estas quince estactones están mas o menos alineadas a lo largo del Golfo (Fig. 1).

Los muestreos se realizaron con botellas Nansen recubiertas con teflón. La temperatura se midió con temómetros reversibles. La salinidad con un salińometro de inducción Bissett-Berman, modelo 6230. El oxlgeno disuelto se determinó por el método micro-Winkler. El pH se determinó con un potenciómetro aigital Ortón, modelo 801, usando electrodos de vidrio de acuerdo con el método descrito por Park (1966a). Para la determinación de alcalintdad total se utilizó el método de Anderson y Robinson (1946) y Park (1966a).

El pH se corrigió por temperatura "in sttu" usando la. ecuación dada por Harvey (1955). Las correcctones se compararon con las calculadas con la ecuactón de Gieskes (1969) y no fueron significativamente diferentes.

La ecuación para el cálculo de la alcalinidad total, tomada de Culberson, Pytckowlez y Hawley (1970) es:

$$
A_{T}=\left(3.0-\frac{130010^{-\mathrm{pH}_{\mathrm{a}}}}{0.74}\right) / \rho(t, s) \quad \mathrm{meq} / \mathrm{Kgr} \text {. }
$$

donde $\mathrm{pH}_{a}$ es el valor del $\mathrm{pH}$ después de agregar el ácido y $\rho$ es la densidad del agua de mar. A partir de lo anterior se calcula la alcalinidad de los carbonatos:

$$
A_{C}=A_{T}-A_{B}
$$

donde $A_{B}$ es la alcalinidad debida a los boratos:

$$
A_{B}=\frac{0.0219 \cdot \mathrm{Cl} \cdot \mathrm{K}_{\mathrm{B}}^{\prime}}{a_{\mathrm{H}}+\mathrm{K}_{\mathrm{B}}^{\prime}} \mathrm{meq} / \mathrm{Kgr} \text {. }
$$

donde $\mathrm{Cl}$ es la clorinidad, $\mathrm{K}_{\mathrm{B}}^{\prime}$ es la constante aparente de disociación del ácido bórico calculada con la ecuactón de Gordon (1973), en base a datos de Lyman (1956) y aH es la actividad del ión hidrógeno.

El bióxido de carbono total se determinó por la ecuación de Skirrow (1965): 


$$
\mathrm{TCO}_{2}=\mathrm{A}_{\mathrm{C}} \frac{\left(\mathrm{a}_{\mathrm{H}}\right)^{2}+\mathrm{a}_{\mathrm{H}} \mathrm{K}_{1}^{\prime}+\mathrm{K}_{1}^{\prime} \mathrm{K}_{2}^{\prime}}{\mathrm{a}_{\mathrm{H}} \mathrm{K}_{1}^{\prime}+2 \mathrm{~K}_{1}^{\prime} \mathrm{K}_{2}^{\prime}} \mathrm{m} \text { Moles } / \mathrm{Kgr} .
$$

donde $K_{1}^{\prime}$ y. $K_{2}^{\prime}$ son la primera y segunda constantes aparentes de disociación del ácido carbónico calculadas con las ecuaciones de Mehrbach, Culberson, Hawley y Pytkowicz (1973), para presión igual a una atmósfera, y corregidas para presión "in situ" con las ecuaciones de Culberson y Pytkowicz (1968).

Para calcular el pH a presión "in situ" se diseño un progra ma de computación, usando el método iterativo sugerido por Ben-Yaakov (1970), El programa se realızó en lenguaje Fortran IV, con una minicomputadora digital Nova 1200 del Centro de Investigactón Cientifica y de Educación Superior de Ensenada, B. C. Este se basa en que el bióxtdo de carbono total no se modifica con los cambios de prestón, sino que sólo cambian sus componentes. Una vez conocido el bióxido de carbono total a una atmósfera de presión, se aplican las ecuactones de Culberson y Pytkowicz (1968) para las correcciones por presión de las

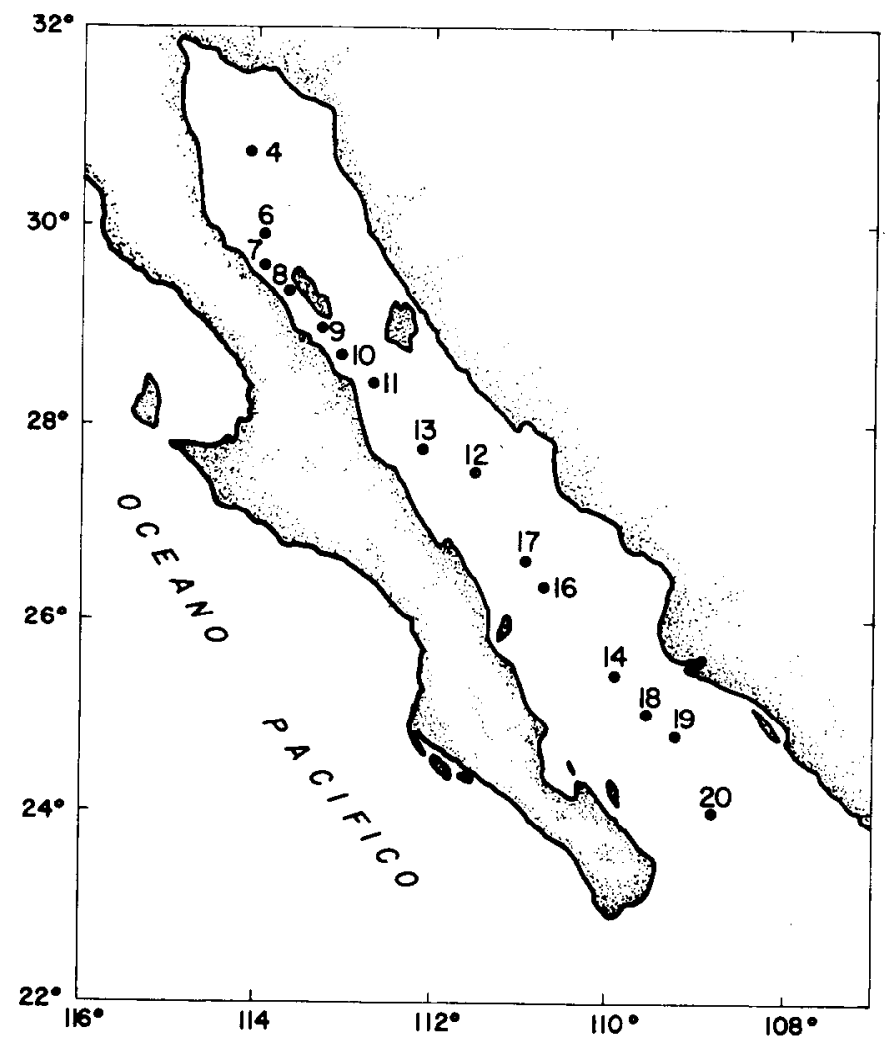

Fig. 1. Localización de las estaciones de muestreo. 
constantes aparentes de disociación del ácido carbónico y del ácido bórico. Se calcula $\mathrm{TCO}_{2}$ con las constantes a presión "in situ" y se ajusta $a_{\mathrm{H}}$ iterativamente de tal manera que $\mathrm{TCO}_{2}$ a presión "in situ" dé prácticamente el mismo valor que $\mathrm{TCO}_{2}$ a una atmósfera.

Con los valores de pH "in situ" se calculd la concentración de carbonato total [ $\mathrm{CO} \overline{\overline{3}}$ ] con la ecuación:

$$
\left[\mathrm{CO}_{3}^{\overline{3}}\right]=\frac{\mathrm{A}_{\mathrm{C}} \cdot \mathrm{K}_{2}^{\prime}}{\mathrm{a}_{\mathrm{H}}+2 \mathrm{~K}_{2}^{\prime}} \quad \mathrm{m} \text { Moles } / \mathrm{Kgr} \text {. }
$$

La concentración de calcio total es dada por Culkin (1965) como una función de la clorinidad:

$$
\left[\mathrm{Ca}^{++}\right]=0.531 \cdot \mathrm{Cl}^{\circ} \% \mathrm{~m} \text { Moles } / \mathrm{Kgr} \text {. }
$$

Los porcientos de saturación "in situ" de carbonato de calcio con respecto a calcita y aragonita se calcularon respectivamente con las ecuaciones:

$$
\begin{aligned}
& \% \text { Sat }_{\mathrm{c}}=\frac{\left[\mathrm{Ca}^{++}\right] \cdot\left[\mathrm{CO}_{3}^{\overline{3}}\right] \cdot 100}{\mathrm{~K}_{\mathrm{Spc}}^{\prime p}} \\
& \% \text { Sat }_{\mathrm{a}}=\frac{\left[\mathrm{Ca}^{++}\right] \cdot\left[\mathrm{CO}_{3}^{\overline{3}}\right] \cdot 100}{\mathrm{~K}_{\mathrm{spa}}^{\prime p}}
\end{aligned}
$$

donde $K_{S p c}^{\prime}$ y $K_{s p a}^{\prime}$ son los productos de solubilidad del carbonato de calcipcon respecto a calcita $y$ aragonita, respectivamente.

Para determinar el producto de solubilidad del carbonato de calcio para calcita, se usaron las ecuaciones de Edmond y Gieskes (1970), con las constantes aparentes de disociación del ácido carbónico de Iyman (1956); y paralelamente las ecuaciones de Ingle (1975), usando las constantes aparentes de disociación del ácido carbónico dadas por Mehrbach, Culberson, Hawley y Pytkowicz (1973). El producto de solubilidad del carbonato de calcio para aragonita se calculo usando las ecuaciones de Edmond $\mathrm{y}$ Gieskes (1970), con las constantes aparentes de disociación del ácido carbónico de Lyman (1956). Las ecuaciones de Edmond y Gieskes (1970) y las de Ingle (1975) se usaron paralelamente en el caso de la calcita, porque existe una discrepancia que no se puede resolver "a priori". 
Las ecuaciones para calcular los productos de solubilidad "in situ" de carbonato de calcio con respecto a calcita y aragonita, dadas por Edmond y Gieskes (1970) son:

$\mathrm{K}_{\mathrm{sp}}^{\prime \mathrm{p}}($ calcita $)=(0.1614+0.02892 \cdot \mathrm{Cl}-0.0063 \cdot \mathrm{t}) \cdot 10\left(\left(-\Delta \mathrm{V}_{\mathrm{c}} \cdot \mathrm{Z}\right) /\left(23.03 \cdot 0.08205 \cdot \mathrm{T} \cdot 10^{3}\right)\right)$

$\mathrm{K}_{\mathrm{sp}(\operatorname{pragonita})}^{\prime p}=(0.5115+0.02892 \cdot \mathrm{Cl}-0.0063 \cdot t) \cdot 10\left(\left(-\Delta \mathrm{V}_{\mathrm{a}} \cdot \mathrm{Z}\right) /\left(23.03 \cdot 0.08205 \cdot \mathrm{T} \cdot 10^{3}\right)\right)$

donde $t$ es la temperatura en grados centígrados, $Z$ es la profundidad en metros, $T$ es la temperatura en grados absolutos $\left({ }^{\circ} \mathrm{K}\right)$, $\Delta V_{C}$ es el incremento del volumen molar por disolución de calcita y $\Delta V_{a}$ para aragonita. $K_{s p}^{\prime}$ esta expresada en $\mathrm{mM}^{2} \cdot \mathrm{Kgr}^{-2}$.

Las ecuaciones para calcular los incrementos del rolumen molar son las siguientes:

$$
\begin{aligned}
& \Delta \mathrm{v}_{\mathrm{c}}=-(35.2-0.20 \cdot \mathrm{t}) \\
& \Delta \mathrm{v}_{\mathrm{a}}=-(33.2-0.20 \cdot \mathrm{t})
\end{aligned}
$$

Estas ecuaciones se obtuvieron con los datos calculados de Edmond y Gieskes (1970) obtenidos en base a datos experimentales de Pytkowicz y Fowler (1967) y Hawley y Pytkowicz (1969), y son una corrección a las ecuaciones presentadas por los primeros autores. Sin embargo, los valores calculados con las ecuaciones de Edmond y Gieskes (1970) caen dentro de los límites de confianza, tomando en consideractón que el error mínimo para $\Delta V$ es de $\pm 1 \mathrm{~cm}^{3} / \mathrm{mol}$.

La ecuación para calcurar el producto de solubilidad de carbonato de calcio con respecto a calcita, a una atmósfera de presión, dada por Ingle (1975) es:

$K_{\mathrm{sp}}^{\prime 1}(S, T)=\left(-34.452-39.866 \cdot \mathrm{S}^{1 / 3}+110.21 \cdot \operatorname{LogS}-7.5752 \cdot 10^{-6} \mathrm{~T}^{2}\right) \cdot 10^{-1}$

donde $S$ es la salinidad. La corrección del producto de solubilidad por cambio de presión es:

$$
\begin{aligned}
& \log \left(K_{s p}^{\prime} p / K_{s p}^{\prime 1}\right)=((48.8-0.53 t)(z-10) / 10+ \\
& \left.+\left(-5.88 \cdot 10^{-3}+1.845 \cdot 10^{-4} t\right)(z-10)^{2} / 100\right) / 188.93(t+273.15)
\end{aligned}
$$


RESULTADOS.

La distribución vertical de temperatura, salinidad y oxígeno disuelto muestra características que han sido reportadas en general por otros autores (Sverdrup, 1941; Roden, 1964) (Fig. 2a, b y c). El umbral sur del canal de Ballenas marca la separación entre dos zonas hidrográficas diferentes. La porción sur del Golfo tiene una distribución espacial de estas propiedades similar a la del Pacifico Oriental Tropical en general. Mientras que la porción norte muestra distribuciones verticales distintas $\mathrm{y}$ únicas. La profundidad máxima del umbral sur del canal de Ballenas o cuenca Salsipuedes es cerca de $450 \mathrm{~m}$ (Rusnak, Fisher y Shepard, 1964), aunque las gráficas aquí presentadas no la muestran. La parte sur presentó en este crucero termoclina, haloclina y oxiclina bien definidas, de la superfice a $10 \mathrm{~s} 200 \mathrm{~m}$ aproximadamente. Mientras que en la parte norte, principalmente en el canal de Ballenas (entre las estaciones $8 \mathrm{y}$ 11), los gradientes verticales fueron mucho menores. Además, en la parte sur existen mínimos de salinidad $\mathrm{y}$ oxígeno a profundidades intermedias, entre 500 y $800 \mathrm{~m}$ aproximadamente; mientras que en la parte norte estas propiedades disminuren monotónicamente con la profundidad. El mínimo de salinidad en la parte sur varía de $34.51 \%$ en la boca del Golfo, a $34.54 \%$ en las cuencas Carmen y Guaymas (estaciones $13 \mathrm{a}$ 16). A $800 \mathrm{~m}$ el canal de Ballenas tiene aproximadamente $5.5^{\circ} \mathrm{C}, 0.3^{\circ} / 00 \mathrm{y} 1.3 \mathrm{ml} / 1$ de $\mathrm{O}_{2}$ más que la parte sur. A 1500 m la diferencia de temperatura es aproximadamente $8^{\circ} \mathrm{C}$. De acuerdo con Roden (1964), la homogeneización en el canal de Ballenas se debe a los fuertes procesos de mezcla provocados principalmente por corrientes de marea. Las propiedades del agua profunda del canal de Ballenas tienden a tener los mismos valores que las de la profundidad del umbral. Esto ocurre en mucho menor grado con las cuencas de la parte sur (Fig. 2a y C). La temperatura aumenta y la salinidad disminuye en las partes profundas, de cuenca a cuenca hacia el norte, además los valores de salinidad son mur homogéneos en cada cuenca: $34.66^{\circ} / 00$ en la cuenca Pescadero (estaciones $19 \mathrm{y} \mathrm{20}$ ), 34.64\% cuenca Faralión (estación 14), 34.63\% en la cuenca Carmen (estación 16), y 34.62\% en la cuenca Guaymas (estación 12). comparadas con $34.68 \%$ en el agua de Fondo del Pacífico fuera del Golfo. El oxígeno también disminuye en las partes profundas, de cuenca a cuenca hacia el norte. A $2500 \mathrm{~m}$ se tIenen: $2.3 \mathrm{ml} / 1$ en Pescadero, $1.7 \mathrm{ml} / 1$ en Farallón $\mathrm{y} 1.4 \mathrm{ml} / 1$ en Carmen.

Los valores mínimos superficiales de $\mathrm{T}^{\circ} \mathrm{C}, \mathrm{S}^{\circ} / \% \mathrm{y} \mathrm{O}_{2}$ se encontraron en el canal de Ballenas, debido también a los procesos de mezcla. Robinson (1973) indicó que las más bajas temperaturas superficiales se encuentran persistentemente en la vecindad de Isla Angel de la Guarda, durante todo el año. 

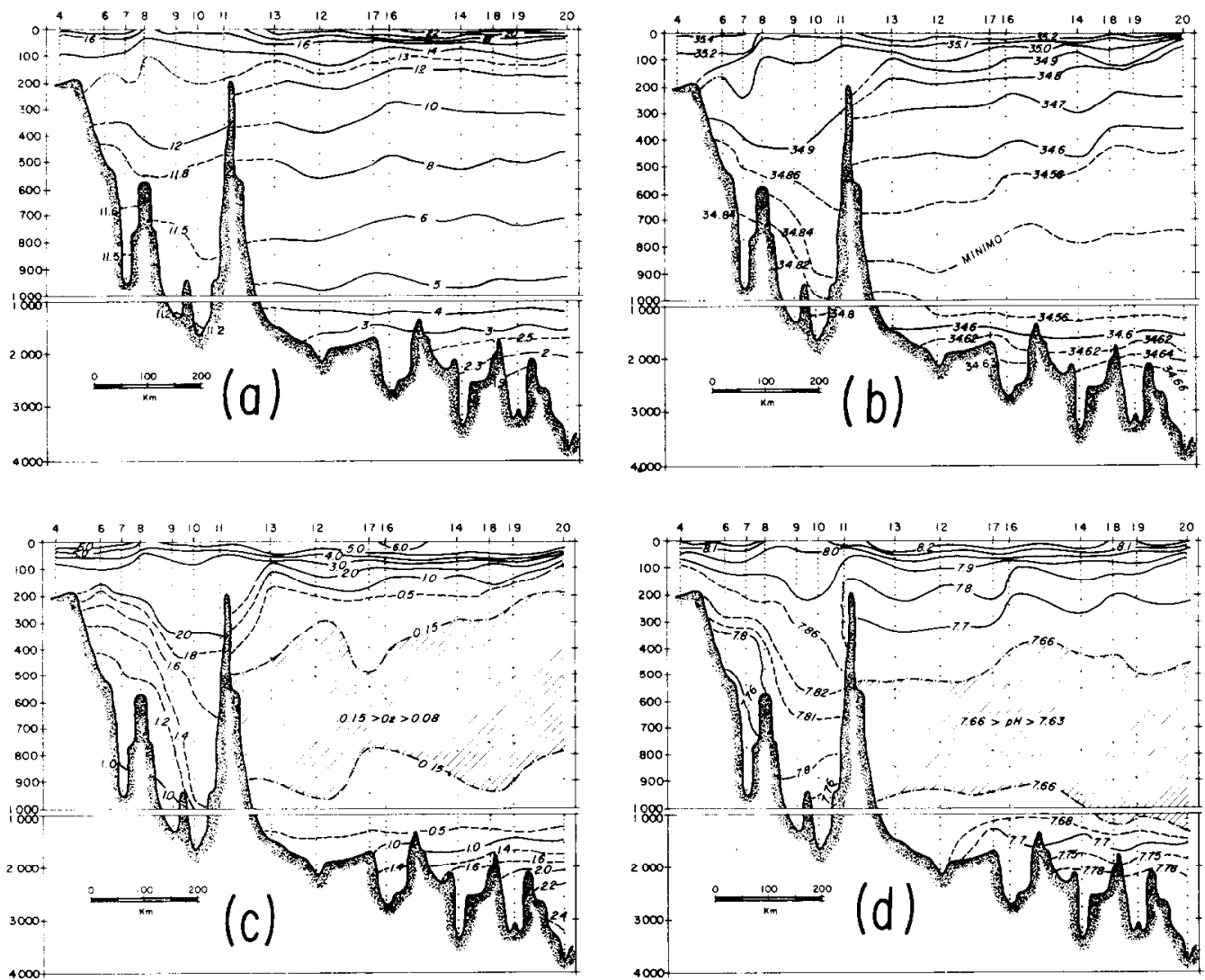

Fig. 2. Distribución vertical de: (a) temperatura $\left({ }^{\circ} \mathrm{C}\right)$;

(b) salinidad $\left(\% / 0_{0}\right)$; (c) oxígeno disuelto $(\mathrm{ml} / \mathrm{l}) ; \mathrm{y}(\mathrm{d}) \mathrm{pH}$. La escala vertical es la profundidad en metros. En la escala horizontal están los números de las estaciones. Los puntos indican las profundidades de muestreo.

La distribución de pH "in situ" concuerda en general con la de $\mathrm{O}_{2}$. Los valores mínimos superficiales, entre 8.0 y 8.1 se encontrarón en el canal de Ballenas (Fig. 2d). En el resto del Golfo los valores superficiales fueron ligeramente mayores que 8.2. En la parte norte el pH disminuyó monotónica mente con la profundidad hasta 7.7 en el fondo de cuenca Salsipuedes (estación 10). A $700 \mathrm{~m}$, en el canal de Ballenas el $\mathrm{pH}$ es aproximadamente 0.18 mayor que en la parte sur. En la parte sur disminuyó con la profundidad hasta un mínimo de 7.63 , aproximadamente a los $700 \mathrm{~m}$, luego aumentó hasta 7.78 a los $2500 \mathrm{~m}, \mathrm{y}$ disminuyó de nuevo hasta 7.72 a $3500 \mathrm{~m}$. Este tipo de máximo profundo de $\mathrm{pH}$ "in situ" fue reportado por Park (1966b) para el Pacífico nororiental, pero a los $3500 \mathrm{~m}$. Se debe a que el efecto de la presión en las constantes de disociación del ácido carbónico, que tiende a disminuir el pH, 
es mayor en altas profundidades que el de disolución de carbonato de calcio y el aumento de oxígeno que tienden a aumentar el $\mathrm{pH}$. La menor profundidad del máximo de $\mathrm{pH}$ reportado en este trabajo, con respecto al reportado por Park (1966b), posiblemente se deba a que nosotros usamos las más nuevas ecuaciones de Culberson y Pitkowicz (1968) para la corrección por presión de las constantes de disoclación.

En la parte norte del Golfo, la alcalinidad total disminuye de $2.35 \mathrm{meq} \mathrm{KBr}^{-1}$ en la superficie a 2.31 meq $\mathrm{Kgr}^{-1}$ a $200 \mathrm{~m}$, y Iuego se mantiene prácticamente constante hasta el fondo $\left(\sim 1500 \mathrm{~m}\right.$ ). En la parte sur disminuye de $2.35 \mathrm{meq} \mathrm{Kgr}^{-1}$ en la superficie a $2.32 \mathrm{meq} \mathrm{Kgr}^{-1}$ aproximadamente a 200-300 m, para luego aumentar a $2.45 \mathrm{meq} \mathrm{Kgr}^{-1}$ en el fondo.

La alcalinidad específica (alcalinidad por unidad de clorinidad) presenta valores cercanos a $0.120 \mathrm{meq} \mathrm{Kgr}^{-1}\left({ }^{\circ} \mathrm{ooo}\right)^{-1}$ en la superficie de todo el Golfo. En la parte norte prácticamente se mantiene este valor para toda la columna de agua. En la parte sur, la alcalinidad específica aumenta monotónicamente hasta alrededor de 0.128 cerca del fondo. En las cuencas de la parte sur, los valores de alcalinidad específica son también prácticamente constantes por debajo de las profundldades de los umbrales, aún para rangos de más de $1500 \mathrm{~m}$ de diferencìa de profundidad entre umbral y fondo.

La concentración superficial de bióxido de carbono total varió entre $2.07 \mathrm{y} 2.15 \mathrm{mM} \cdot \mathrm{Kgr}^{-1}$, con los valores mayores en el canal de Ballenas, de acuerdo con la distribución de oxígeno (Fig. 3a). En la parte sur, el bióxido de carbono total aumenta monotónicamente con la profundidad, con valores de $2.40 \mathrm{mM} \cdot \mathrm{Kgr}^{-1}$ en la parte profunda de la cuenca de Guaymas (estación 16). En la boca del Golfo los valores son casi constantes entre 850 y $3500 \mathrm{~m}$; el incremento es sólo $0.05 \mathrm{mM} \cdot \mathrm{Kgr}^{-1}$ en este rango de profundidad. En la parte norte, aumenta monotónicamente con la profundidad, con un gradiente mucho menor que en la parte sur. De los 200-300 m hasta el fondo de cuenca Salsipuedes (estactón 10) los valores son prácticamente constantes. A $1000 \mathrm{~m}$, en el canal de Ballenas el bióxido de carbono total es $0.1 \mathrm{mM} \cdot \mathrm{Kgr}^{-1}$ menor que en la parte sur.

Los valores superficiales del porciento de saturación de carbonato de calcio con respecto a aragonita varían aproximada mente entre 150\% y $250 \%$, con valores mínimos en el canal de Ballenas (Fig. 3b). En la parte sur existe un gradiente vertical mur marcado de la superficie a 100-150 m. La isograma de $100 \%$ se encuentra aproximadamente a $50-150 \mathrm{~m}$ a 10 largo de todo el Golfo, tendiendo a ser IIgeramente más somera en la 
boca que en el extremo interno. Los valores iisminuyen monotónicamente con Ia profundidad en la parte sur hasta $40 \%$ aproximadamente a $1000 \mathrm{~m}$, manteniéndose prácticamente constante a profundidades mayores. En la parte norte el gradiente vertical cerca de la superficie es mucho menor que en la parte sur, la

variación es monotónica con valores de $60 \%$ a 1000-1500 m. A $1000 \mathrm{~m}$, en el canal de Ballenas el porciento de saturación de aragonita es cerca de 20 unidades mayor que en la parte sur.

El porciento de saturación de carbonato de calcio con respecto a calcita, calculado con las ecuaciones de Edmond y Giekes (1970), presenta valores superficiales aproximadamente entre $200 \%$ y $400 \%$, con valores minimos en el canal de Ballenas (Fig. 3c). Los valores disminuyen monotónicamente con la profundidad, En la parte sur hay un fuerte gradiente en Ios primeros $100 \mathrm{~m}, \mathrm{y}$ la isograma de $100 \%$ se encuentra entre 150 y $300 \mathrm{~m}$; por debajo de los $1500 \mathrm{~m}$ los valores son prácticamente constantes e igual a $60 \%$. En la parte norte el gradiente vertical es menor, con la isograma de $100 \%$ a $800-1000 \mathrm{~m}$, y valores de $85 \%$ a $1500 \mathrm{~m}$. A $1000 \mathrm{~m}$, en el canal de Ballenas los valores son 30 unidades mayores que en la parte sur.

Los porcientos de saturación de carbonato de calcio con respecto a calcita calculados con las ecuaciones de Ingle (1975), son mayores que los calculados con las ecuaciones de Edmond y Gieskes (1970) (Fig. 3d). Los valores superficiales son aproximadamente entre $300 \%$ y $500 \%$, con valores mínimos en el canal de Ballenas. En la parte sur se presenta un gradiente vertical fuerte en los primeros $100 \mathrm{~m}$. Los valores disminuyen monotónicamente hasta cerca de $60 \%$ en el fondo de cuenca Pescadero (estación 20). La isograma de $100 \%$ se encuentra entre 350 y $600 \mathrm{~m}$. En la parte norte el gradiente vertical es más débil. El 100\% se encuentra hasta aproximadamente $1200 \mathrm{~m}$ en cuenca Salsipuedes (estación 10). Al fondo de la misma los valores son cercanos a 90\%. A $1000 \mathrm{~m}$ los valores son 30 unidades de porciento mayores en cuenca Salsipuedes que en la parte sur.

\section{DISCUSIONES.}

Las temperaturas superficiales en el Golfo presentan un gradiente con los valores ascendiendo en general de la boca al extremo interno, de octubre a junio; mientras que de julio a septiembre la distribución superficial de temperatura es relativamente homogénea, conservándose durante todo el año valores mínimos en la vecindad de isla Angel de la Guarda (Robinson, 1973). De cuardo con Alvarez Borrego, Rivera, Gaxiola Castro, Acosta Ruiz y Schwartzlose (1978), en la parte sur del Golfo, 

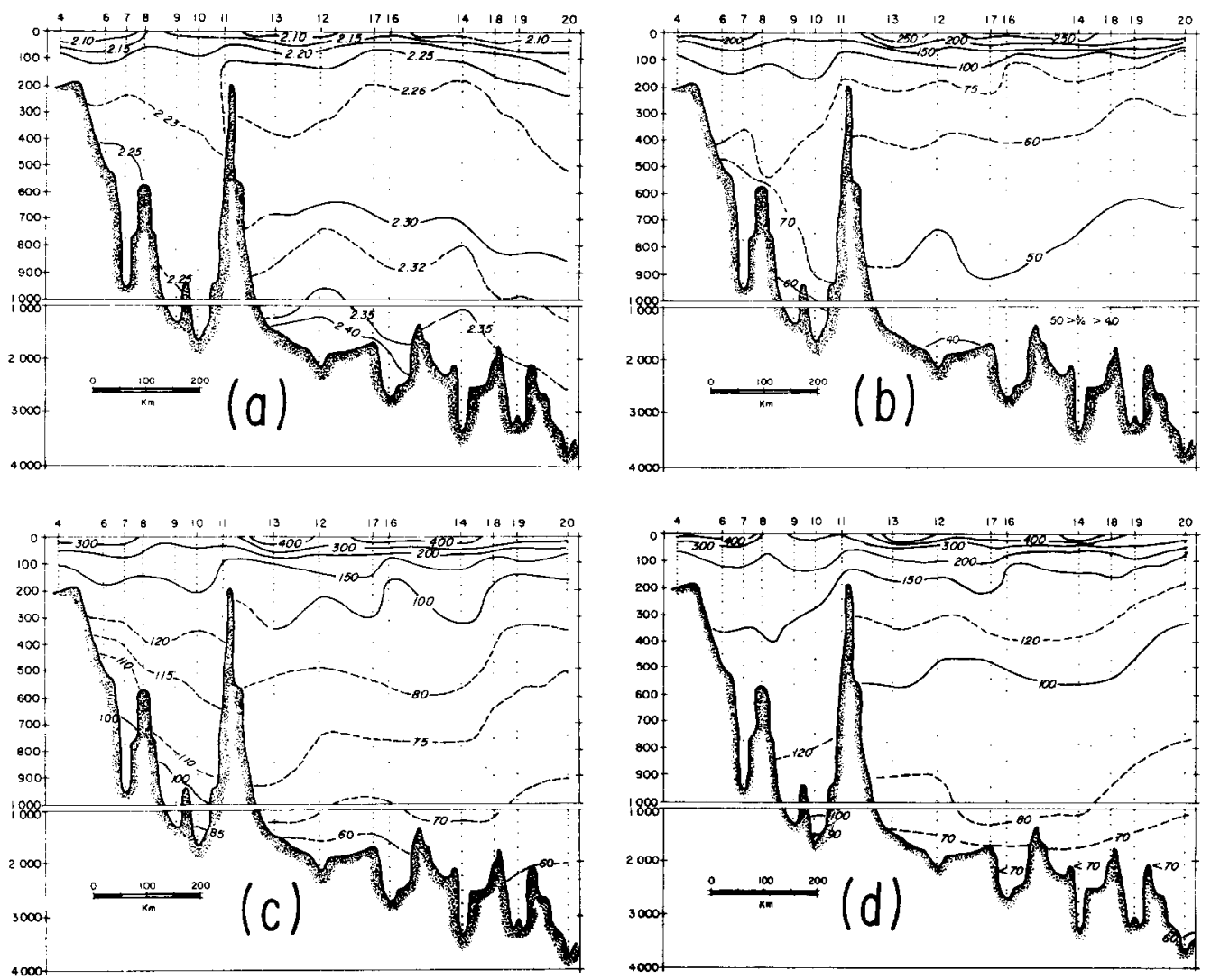

Fig. 3. Distribución vertical de: (a) bióxido de carbono inorgánico total ( $\mathrm{mM} \mathrm{Kgr}^{-1}$ ); (b) porciento de saturación de carbonato de calcio con respecto a aragonita; (c) porciento de saturación con respec to a calcita calculado con las ecuaciones de Edmond y Gieskes (1970); y (d) porciento de saturación con respecto a calcita calculado con las ecuaciones de Ingle (1975).

estos cambios estacionales afectan la distribuctón de temperatura hasta los $300 \mathrm{~m}$ aproximadamente. Las mayores temperaturas superficiales de la parte sur del Golfo, registradas en nuestro crucero, son la causa de los mayores valores superficiales del porciento de saturación de carbonato de calcio en el sur que en el norte. En verano, los valores superficiales del porciento de saturación deben ser mayores y más homogéneos. Los mínimos valores superficiales de porciento de saturación encontrados en el canal de Ballenas se deben al efecto combinado de mínimas temperaturas y $\mathrm{pH}$, que a su vez se deben al efecto de mezcla por las fuertes corrientes de marea. Los elevados valores de porciento de saturación de carbonato de calcio en la superficie se deben a que la precipitación inorgánica del mismo se inhibe fuertemente en el agua de mar por el ión magnesio y por la materia orgánica disuelta (Pytkowicz, 1965; Suess, 1970; citados por Edmond, 1974). 
El mínimo de salinidad a profundidades intermedias (700 $900 \mathrm{~m}$ ), reportado por primera vez por Sverdrup (1941), es el núcleo del Agua Intermedia del Antártico (Griffiths, 1968). Ilega hasta la parte norte de la cuenca de Guaymas, un poco al norte de $28^{\circ} \mathrm{N}$. Bajo ella se encuentra el Agua del Fondo del Pacífico proveniente del Antártico.

La constancia de alcalinidad específica en las cuencas, por debajo de los umbrales, puede indicar muy poca disolución de carbonato de calcio o la presencia de procesos de mezcla suficientemente fuertes para abatir los gradientes que se pudieran formar por disolución. La mayor profundidad de la isograma de $100 \%$ en el caso de calcita, y muy levemente en el caso de aragonita, en la parte norte con respecto a la parte sur, se debe principalmente a que en el norte hay mayores valores de temperatura $\mathrm{y}$ pH que en el sur, para una determinada profundidad.

Los valores de porciento de saturación de carbonato de calcio con respecto a calcita calculados con las ecuaciones de Ingle (1975) fueron aproximadamente 1.2 multiplicado por los valores calculados con las ecuaciones de Edmond y Gieskes (1970). Gieskes (1974) indica que los valores experimentales de Duedall (1972), para el incremento del volumen molar de carbonato de calcio por disolución $(\Delta \bar{V})$, son diferentes a los calculados por Edmond y Gieskes (1970) y expresa que se requieren más datos experimentales sobre la dependencia de la solubilidad de carbonato de calcio con respecto a presión, para rangos más amplios de temperatura y salinidad. Los valores de Duedall (1272) a $25^{\circ} \mathrm{C}$ y $35^{\circ} \%$ son $\Delta \overline{\mathrm{V}}_{\mathrm{g}}=-41.8 \mathrm{~cm}^{3} \mathrm{~mol}^{-1}$ y $\Delta \bar{\nabla}_{\mathrm{a}}=$ $-39.1 \mathrm{~cm}^{3} \mathrm{~mol}^{-1}$. Con estos vaiores $\mathrm{y}$ utilizando la misma dependencia con respecto a temperatura determinada por Hawley y Pytkowicz (1969), habría que multiplicar los valores calculados de porciento de saturación de carbonato de calcio con las ecuaciones de Edmond y Gieskes (1970) por los siguientes factores: a $0 \mathrm{~m}, 100$; a $1000 \mathrm{~m}, 0.95$; a 1500 $\mathrm{m}, 0.93$; a $2000 \mathrm{~m}, 0.90$; y a $3000 \mathrm{~m}, 0.86$; en ambos casos, el de calcita y el de aragonita. Esto prácticamente no cambiaría la posición de las isogramas de $100 \%$ en la parte sur del Golfo. En la parte norte la subiría unos $100 \mathrm{~m}$ en el caso de la calcita. A $3000 \mathrm{~m}$, en el sur, el porciento con respecto a aragonita bajaría a $37 \%$, y el de calcita a $52 \%$. Por supuesto, ésto aleja los valores todavía más de los calculados con las ecuaciones de Ingle (1975).

De acuerdo con van Andel (1964), los sedimentos del Golfo contienen carbonato de calcio en toda su extensión, aún en las partes mas profundas. Pytkowicz (1970) indica que el horizonte de saturación, o isograma de $100 \%$ de calcita, está a menor profundidad que el horizonte de compensación. Este 
último está en la profundidad en la que el arribo de exoesqueletos calcáreos de aguas superiores se compensa por la disolución. De acuerdo con Edmond (1974), en el Pacífico norte el horizonte de compensación está aproximadamente a $3900 \mathrm{~m}$. Arriba de este horizonte los sedimentos contienen carbonato de calcio relativamente abundante a pesar de que la columna de agua tiene valores de porciento de saturación menores a 100\%. Esto se debe a una velocidad de arribo de exoesqueletos calcáreos mayor que la velocidad de disolución.

\section{RECONOCIMIENTOS.}

Se agradece al personal del Centro de Cómputo del CICESE su valiosa ayuda en la elaboración de los programas y en la operación de la computadora. Nuestro más sincero reconocimiento a todos los participantes del crucero del "B/I Alexander Agassiz" al Golfo de California, en abril-mayo de 1974.

\section{BIBLIOGRAFIA.}

Alvarez Borrego, S., J. A. Rivera, G. Gaxiola Castro, M. J. Acosta Ruiz y R. A. Schwartzlose, 1978. Nutrientes en el Golfo de California. Ciencias Marinas (Mex), ésta publicación.

Anderson, D. H. and R. J. Robinson. 1946. Rapid electrometric determination of the alkalinity of seawater using a glass electrode, Ind. Eng. Chem., Anal. Ed. 18:767-773.

Been-Yaakov, S. 1970. A method for calculating the in situ pH of seawater. Limnol. \& Oceanogr. 15:326-328.

Culberson, C. H. 1972. Processes affecting the oceanic distri bution of carbon dioxide. Ph. D. thesis. School of Oceanography, Oregon State University. Corvallis, Oregon. 178 p.

Culberson, C. and R. M. Pytkowicz. 1968. Effect of pressure on carbonic acid, boric acid, and the $\mathrm{pH}$ in seawater. Limnol. \& Oceanogr. 13:403-417.

Culberson, C., R. M. Pytkowicz and J. E. Hawley. 1970. Seawater alkalinity determination by the $\mathrm{pH}$ method. J. Mar. Res. $28: 15-21$.

Culkin, F. 1965. The major constituents of seawater. In Chemical Oceanography, Vol. 1. Ed. J. P. Riley and G. Skirrow. Academic Press, New York: 121-161. 
Duedall, I. W. 1972. The partial molal volume of calcium carbonate in seawater. Geochim. Cosmochim. Acta. 36:729-734.

Edmond, J. M. 1974. On the dissolution of carbonate and silicate in the deep ocean. Deep-Sea Res. 21:455-480.

Edmond, J. M. and J. M. Gieskes. 1970. On the calculation of the degree of saturation of seawater with respect to calcium carbonate under in situ conditions. Geochim. Cosmochim. Acta. 34:1261-1291.

Gieskes, J. M. 1969. Effect of temperature on the pH of seawater. Limnol. Oceanogr. 14:679-685.

Gieskes, J. M. 1974. The alkalinity-total carbon dioxide system in seawater. In: The Sea, Vol. 5: Marine Chemistry. E. D. Goldberg (ed.). J. Wiley \& Sons. 123-152.

Gordon, L. I. 1973. A study of carbon dioxide partial pressures in surface waters of the Pacific ocean. Ph. D. thesis. School of Oceanography. Oregon State University. Corvallis, Oregon. 216. p.

Griffits, R. G. 1968. Physical, Chemical and biological oceanagraphy of the entrance to the Gulf of California, Spring of 1960. Spec. Scient. Rep.-Fish., U. S. Fish Wildl. Serv. $47 \mathrm{p}$.

Harvey, H. W. 1955. The chemistry and fertility of seawaters. Cambridge Univ. Press. 224 p.

Hawley, J. and R. M. Pytkowicz, 1969. Solubility of calcium carbonate in seawater at high pressure and $2^{\circ} \mathrm{C}$. Geochim. Cosmochim. Acta, 33:1557-1561.

Ingle, S. E. 1975. Solubility of calcita in the ocean. Marine Chemistry, 3:301-319.

Lyman, J. 1956. Buffer mechanism of seawater. $\mathrm{Ph}$. D. thesis. Univ. Calif., Los Angeles..196. p.

Mehrbach, C., C. H. Culberson, J. E. Hawley and R. M. Pytkowicz. 1973. Measurement of the apparent dissociation constants of carbonic acid in seawater at atmospheric pressure. Limnol. \& Oceanogr. 18: 897-907.

Park, P. K. 1966a. Surface pH of the northeastern Pacific Ocean. J. Oceanol. Soc. Korea 1:1-6.

Park, P. K. 1966b. Deep-sea pH. Science 154(3756):1540-1542. 
Pytkowicz, R. M. 1970. On the carbonate compensation depth in the Pacific Ocean. Geochim Cosmochim. Acta. 34:836-839.

Pytkowicz, R. M. and G. A. Fowler. 1967. Solubility of foraminifera in sea water at high pressures. Geochem. J. 1: $525-570$.

Roden, G. I. 1964. Oceanographic aspects of the Gulf of California. In: Marine Geology of the Gulf of California, a Symposium. van Andel Ty. H. and G. G. Shor Jr. (Eds.). Amer. Assoc. Petrol. Geol., Memoir 3:30-58.

Robinson, M. K. 1973. Atlas of monthly mean sea surface and subsurface temperatures in the Gulf of California, Mexico. San Diego Soc. Nat. Hist. Memoir 5.97 p.

Rusnak, G. A., R. L. Fisher and F. P. Shepard. 1964. Bathymetry and faults of Gulf of California. In: Marine Geology of the Gulf of California., a Symposium. van Andel Ty. H. and G. G. Shor Jr. (Eds.). Amer. Assoc. Petrol. Geol., Memoir 3: 59-75.

Skirrow, G. 1965. The dissolved gases-carbon dioxide. In: Chemical Oceanography, Vol. I Ed. J. P. Riley and G. Skirrow. Academic Press, New York: 227-322.

Sverdrup, H. U. 1941. The Gulf of California: Preliminary discussion on the cruise of the "E. W. Scripps" in February and March, 1939. 6th. Pac. Sci. Cong. Proc. 3: $161-166$.

van Andel, Tj. H. 1964. Recent marine sediments of the Gulf of California. In: Marine Geology of the Gulf of California, a Symposium. van Andel Tj. H. and G. G. Shor Jr. (Eds.). Amer. Assoc. Petrol. Geol., Memoir 3:216-310.

Recibido: 17 de noviembre de 1978. 